\title{
EXISTENTIALLY COMPLETE ABELIAN LATTICE-ORDERED GROUPS \\ BY
}

\author{
A. M. W. GLASS AND KEITH R. PIERCE
}

\begin{abstract}
The theory of abelian totally ordered groups has a model completion. We show that the theory of abelian lattice-ordered groups has no model companion. Indeed, the Archimedean property can be captured by a first order $\forall \exists \forall$ sentence for existentially complete abelian lattice-ordered groups, and distinguishes between finitely generic abelian lattice-ordered groups and infinitely generic ones. We then construct (by sheaf techniques) the model companions of certain classes of discrete abelian lattice-ordered groups.
\end{abstract}

The class of abelian groups has a model companion [7]. The slightly larger class of nilpotent class 2 groups, however, is already very misbehaved; there is no model companion and, indeed, the finitely generic and infinitely generic theories share no models in common ([17] and [18]). It is natural to wonder, therefore, what happens if, instead of enlarging the class of groups, we enlarge the language by adding a binary relation $\leqslant$ compatible with the group operation (cf. passing from fields to ordered fields). A. Robinson has shown the following:

THEOREM A [15, p. 36]. The theory of abelian totally ordered groups has a model completion; viz. the theory of divisible abelian totally ordered groups having at least two different elements.

When total order is replaced by lattice-order, the conclusion changes radically. Our main theorem is:

THEOREM B. (1) The theory of abelian lattice-ordered groups has no model companion. Moreover,

(2) the Archimedean property is equivalent to a $\forall \exists \forall$ sentence for existentially complete abelian lattice-ordered groups, and

(3) finitely generic abelian lattice-ordered groups satisfy the sentence (i.e., are Archimedean) but infinitely generic ones do not (i.e., are not Archimedean).

Of course, the Archimedean property is not expressible by a first order sentence in general; it is only when we restrict to existentially complete abelian latticeordered groups that we can capture it by a first order sentence. This expressibility phenomenon is frequently used to distinguish between finitely generic structures

Received by the editors April 6, 1979 and, in revised form, October 12, 1979.

AMS (MOS) subject classifications (1970). Primary 03C25, 03C60, 06F20.

Key words and phrases. Abelian lattice-ordered groups, model companion, model completion, existentially complete, algebraically closed, finite forcing, infinite forcing, sheaf, section. 
and infinitely generic ones (see [10], [13], [17] and [18]). Moreover, a $\forall \exists \forall$ sentence is the simplest that can ever be used to distinguish between such structures (see, e.g., [9, Appendix, Proposition 24]).

Let $T$ be a consistent theory. Let $\mathbf{R} T$ denote the theory obtained from taking subdirect products of models of $T$ (i.e., $\mathbf{R} T$ is the set of sentences which hold in all models which are subdirect products of models of $T$ ). If $T$ has a set of universal axioms, $\mathbf{R} T=T_{\mathrm{UH}}$ the universal Horn theory obtained from $T$ [20]. William H. Wheeler [20] has conjectured that if $T$ is a consistent theory having a set of universal axioms, then $T_{\mathrm{UH}}$ has a model companion if $T$ does. But if we let $T$ be the theory of abelian totally ordered groups (in the language with the group and lattice operations), $T_{\mathrm{UH}}$ is the theory of abelian lattice-ordered groups (see the remarks following the proof of Theorem A). Theorems A and B therefore refute the Wheeler conjecture:

COROllary C. There is a consistent theory $T$ having a finite set of positive universal axioms such that $T$ has a model completion but $T_{\mathrm{UH}}$ has no model companion.

We confess to being baffled as to when the existence of a model companion (for a universal theory) carries over to the subdirect product theory. Our counterexample involves order. If order is playing an essential role, one might avoid it by considering only stable theories. Thus a natural conjecture is: If $T$ is a stable universally axiomatizable theory, then $T_{\mathrm{UH}}(=\mathrm{R} T)$ has a model companion if $T$ does. $^{1}$

In the last part of this paper we establish model companions for certain classes $\mathcal{K}$ of discrete abelian totally ordered groups and for the corresponding classes $\mathbf{R} \mathcal{K}$ of discrete abelian lattice-ordered groups $(\mathcal{K}=\operatorname{Mod}(\mathcal{T})$ for some theory $\mathscr{T})$. This indicates that if our conjecture is true, stability, though sufficient, is not necessary to pass from $T$ to $T_{\mathrm{UH}}$ (for existence of model companions).

We have assumed that Theorem $B$ would have a larger audience than other portions of this paper which are more technical. For this reason we have relegated the technical results to the end so that the supposed general reader can obtain an uncluttered self-contained proof of Theorem B by reading only the first half of this paper. In order to make our results accessible to algebraists and model theorists alike, there will be portions that each can (and should!) easily skip over.

Recall that if $T$ is a theory and $\mathfrak{A}$ is a submodel of a model of $T$, then $\mathfrak{A}$ is said to be existentially complete in $T$, or $T$-existentially complete, if any existential sentence of $\mathcal{L}(\mathfrak{U})$ (the language $\mathfrak{E}$ augmented by constants naming the elements of $\mathfrak{U}$ ) which is true in a model of $T$ including $\mathfrak{A}$, is already true in $\mathfrak{A}$. If instead of considering all existential sentences of $\mathfrak{L}(\mathfrak{A})$, we restrict to those obtained by existentially quantifying over conjunctions of atomic sentences, we call the corresponding structures algebraically closed in $T$. In the presence of the group axioms, algebraically closed corresponds to having a solution to $w_{0}(\mathbf{x}, \mathbf{a})=0, \ldots, w_{n-1}(\mathbf{x}, \mathbf{a})=0$

\footnotetext{
${ }^{1}$ However, Mary Anne Evans' beautifully expressed warning should also be heeded: "But this power of generalising which gives men so much the superiority in mistake over the dumb animals, ... " [8].
} 
in $\mathfrak{A}$ whenever there is a solution in some group $\mathfrak{B} \supseteq \mathfrak{A}$, and existentially complete allows also $w_{n}(\mathbf{x}, \mathbf{a}) \neq 0, \ldots, w_{m}(\mathbf{x}, \mathbf{a}) \neq 0(\mathbf{a} \in \mathfrak{U})$. For the theory of fields, the concepts coincide $(w \neq 0$ can be replaced by solving wy $-1=0)$ and yield the standard algebraically closed fields. For abelian torsion-free groups, the concepts coincide and yield the class of divisible torsion-free abelian groups (except that $\{0\}$ is algebraically closed but not existentially complete).

A theory $T$ has a model companion theory $T^{c}$ if and only if:

(1) every model of $T$ is contained in a model of $T^{c}$ and conversely, and

(2) $T^{c}$ is model-complete; i.e., every model of $T^{c}$ is existentially complete in $T^{c}$.

If it exists, the model companion is unique. It is easy to see that every (infinite) model of $T$ is contained in a $T$-existentially complete model (of the same cardinality, if $\mathcal{E}$ is countable). Consequently, if the $T$-existentially complete structures form an elementary class (i.e., there is a set of sentences $S$ whose models are precisely the $T$-existentially complete structures), then this class yields the model companion; if it is not an elementary class, the model companion does not exist. In the presence of the amalgamation property, the model companion is the model completion, in which the relationship between $T$ and $T^{c}$ is even stronger.

Note that, since existential quantifiers distribute over disjunctions, in any proof of model-completeness it suffices to consider only primitive formulas (i.e., existential formulas whose quantifier-free part is a conjunction of atomic and negated atomic formulas).

Special cases of existentially complete structures are the finitely generic and infinitely generic ones. The infinitely generic ones are large in the sense that if $\mathfrak{U}$ is a model of $T$, there is an infinitely generic structure $\mathfrak{B}$ containing $\mathfrak{A}$ as a submodel. If $T$ enjoys the joint embedding property (the theory of abelian lattice-ordered groups does since the direct sum of two abelian lattice-ordered groups is an abelian lattice-ordered group), any two infinitely generic structures satisfy the same first order sentences. This is all we will need about them. The finitely generic structures on the other hand are small in the sense that if $\mathfrak{A} \subseteq \mathfrak{B}, \mathfrak{A}$ is existentially complete in $T$ and $\mathfrak{B}$ is finitely generic, then $\mathfrak{A}$ is finitely generic. This is why we might expect "Archimedean" (if we could capture it first order for existentially complete abelian lattice-ordered groups) to hold in finitely generic abelian lattice-ordered groups but fail in the infinitely generic ones. Since our language is countable and our theory enjoys the joint embedding property, we have that the finitely generic structures are among the $T$-existentially complete structures that satisfy the complete theory $T^{f}$. ( $T^{f}$ is the set of sentences $\phi$ of $\mathcal{L}$ such that $\varnothing \Vdash \neg \neg \phi$. Let $C$ be a countable set of constants not occurring in $\mathcal{L}$. A finite set $p$ of basic sentences (atomic or negated atomic) of $\mathcal{L}(C)$ is called a condition if $T \cup p$ is consistent. If $p$ is a condition and $\phi$ is a sentence of $\mathcal{L}(C), p \Vdash \phi .(p$ forces $\phi)$ is defined inductively: for $\phi$ atomic, if $\phi \in p$; for $\&$, or, $\exists$ do the obvious; but $p \Vdash \neg \psi$ if no condition $q \supseteq p$ forces $\psi$.)

A final comment on notation: we shall use the same symbol to denote an elementary class and the first order theory of that class.

If this review has been inadequate, see the appendix (The Lazy Algebraist's Guide to Model-theoretic Forcing) to our article [9], G. Cherlin's book [2], or J. 
Hirschfeld and W. H. Wheeler's book [10]. This last is very complete and thorough.

1. Proof of Theorem B. Let $G$ be an abelian group and $\leqslant$ a partial order on $G$. $G$ is a partially ordered group (with respect to $\leqslant$ ) if $G$ satisfies:

$$
\forall x \forall y \forall z(x \leqslant y \rightarrow x+z \leqslant y+z) .
$$

If $G$ is a partially ordered group and $\leqslant$ is a lattice (total) order, then $G$ is said to be a lattice-ordered group (totally ordered group) or l-group (o-group). The monotonicity of the group operation implies that it distributes over the lattice operations:

$$
(x \vee y)+z=(x+z) \vee(y+z)
$$

and dually. If $G$ is an $l$-group, the lattice is distributive.

We will use $\vee$ and $\wedge$ for the supremum and infimum (lattice) operations and reserve or, $W$ and $\&, M$ for disjunctions and conjunctions respectively. The following facts are part of the folklore of the subject (Lemmas 1 and 2 do not need the abelian hypothesis).

LEMMA 1. If $G$ is an abelian l-group, then $G$ is torsion-free.

Proof. A straightforward induction shows

$$
n(g \vee 0)=n g \vee(n-1) g \vee \cdots \vee 0 .
$$

Hence $n g=0$ implies

$$
n(g \vee 0)=(n-1) g \vee \cdots \vee 0=(n-1)(g \vee 0) .
$$

Thus $g \vee 0=0$. Similarly, $g \wedge 0=0$ so $g=0$.

LEMMA 2. If $G$ is an abelian l-group and $g \wedge f=0$, then $g \wedge n f=0$ for all positive integers $n$.

Proof. By induction on $n$. If $x=g \wedge(n+1) f$, then $x \geqslant 0$ and $x-f \leqslant g$, nf so $x-f \leqslant 0$. Hence $x \leqslant f, g$ so $x \leqslant 0$.

LEMMA 3. If $G$ is an abelian l-group (o-group), then there is a unique way to extend the order on $G$ to $\bar{G}$, the divisible closure of $G$. With this inherited order, $\bar{G}$ is an l-group (o-group).

Proof. For each $x \in \bar{G}$, there is a positive integer $n$ such that $n x \in G$. Define $x \geqslant 0$ in $\bar{G}$ if and only if $n x \geqslant 0$ in $G$, and $x \geqslant y$ in $\bar{G}$ if and only if $x-y \geqslant 0$ (in $\bar{G})$. It is easily verified that this order fulfills the promises of the lemma.

For the sake of completeness, and because of its simplicity, we include a sketch of the proof of Theorem A. Since the two theories are model consistent and since the theory of abelian totally ordered groups has the amalgamation property [14], it suffices to prove model completeness of the theory of divisible abelian totally ordered groups. Since all abelian lattice-ordered groups are torsion-free; then this theory can be transformed into a universal theory by adjoining to the language unary functions, one for each rational number $q$, whose actions are multiplication by these rationals. The expert model theorist knows that, in a universal theory, the proof of model completeness can be reduced to the consideration of existential formulae of one quantified variable. Therefore it suffices to prove: If $A$ and $B$ are 
divisible abelian totally ordered groups, $A \subseteq B, \mathbf{a} \in A$, and $\exists x \phi(x, \mathbf{a})$ is a primitive formula satisfiable in $B$, then it is satisfiable in $A$.

Observe that, in totally ordered groups, lattice operations are trivial, so nothing changes if we express $\phi$ in terms of equations and inequalities. Using the usual laws for inequalities, we can assume that $\phi$ is a conjunction of disjunctions of equations and inequalities of one of the forms $x \leqslant a, a \leqslant x, x<a, a<x, x=a$ (note that the new unary functions allow operations like replacing $2 x \leqslant a$ by $x \leqslant a / 2$ ) and therefore the set of all solutions, in $B$, of $\phi(x, a)$ is simply an interval with endpoints in $A$. Since $A$ is divisible, it is dense, and therefore, the nonemptiness of this solution set in $B$ implies its nonemptiness in $A$. Therefore $\phi(x, a)$ is satisfiable in $A$. This completes the sketch of the proof of Theorem A.

If $G, H$ are abelian $l$-groups, $G \oplus H$ will always get the order: $\left(g_{1}, h_{1}\right) \geqslant\left(g_{2}, h_{2}\right)$ if and only if $g_{1} \geqslant g_{2}$ (in $G$ ) and $h_{1} \geqslant h_{2}$ (in $H$ ), unless otherwise stated. $G \oplus H$ is then an abelian $l$-group. We order $\sum_{i \in I} G_{i}$ similarly, even when $I$ is infinite. If each $G_{i}$ is an abelian $l$-group, so is $\sum_{i \in I} G_{i}$, the $\vee$ and $\wedge$ operations being pointwise.

Let $G$ be an abelian lattice-ordered group. A subgroup $M$ of $G$ which is also a sublattice is called an l-subgroup. $M$ is convex if $a \leqslant x \leqslant b$ and $a, b \in M$ imply $x \in M$. The convex $l$-subgroups of $G$ (called solid subgroups by Bigard et al. [1]) are precisely the kernels of homomorphisms from $G$.

A convex $l$-subgroup $P$ of $G$ is said to be prime if $G / P$ is a totally ordered group (in the inherited order). Prime subgroups abound. One way to obtain them is as follows: Let $0 \neq g \in G$. Then any convex $l$-subgroup which is maximal with respect to not containing $g$ (existence by Zorn's lemma) is a prime subgroup. If we choose one such prime subgroup, $P_{g}$, for each $0 \neq g \in G$, we obtain a map of $G$ into $\Pi\left\{G / P_{g}: 0 \neq g \in G\right\}$. Each $G / P_{g}$ is an abelian totally ordered group and since $g \notin P_{g}$, this map is an embedding. Hence $G$ is realized as a subdirect product of abelian totally ordered groups. The proof of Corollary $\mathrm{C}$ now follows once we have proved Theorem B. If $G$ is an $l$-subgroup of an abelian lattice-ordered group $H$, then $G \cap Q$ is a prime subgroup of $G$ whenever $Q$ is a prime subgroup of $H$; moreover, all prime subgroups of $G$ are obtained in this way. For details, and for other basic facts about lattice-ordered groups, see [1].

Let $\mathcal{Q}$ be the class of abelian l-groups.

Proposition 4. If $G \in \mathbb{Q}$ is a direct sum of divisible abelian o-groups, then $G$ is algebraically closed in $\mathbb{Q}$, but not existentially complete in $\mathbb{Q}$.

Proof. Let $G=\sum_{i \in I} G_{i}$ with each $G_{i}$ a divisible o-group. Let $P_{j}=\sum_{i \neq j} G_{i}$. Clearly $P_{j}$ is prime $\left(G / P_{j} \cong G_{j}\right)$. Let $\phi(\mathbf{x}, \mathbf{g})$ be a conjunction of atomic formulae in $\mathcal{L}(G)$, with $\mathbf{g} \in G$ and let $G \subseteq H \in \mathbb{Q}$ so that $H \vDash \phi(\mathbf{h}, \mathbf{g})$. By the introductory remarks, there are prime subgroups $Q_{j}$ of $H$ such that $P_{j}=G \cap Q_{j}$. But $G / P_{j}$ is canonically embedded in $H / Q_{j}$ via the second isomorphism theorem $\left(G / P_{j}=\right.$ $G / Q_{j} \cap G \cong\left\langle G, Q_{j}\right\rangle / Q_{j} \subseteq H / Q_{j}$ ) and by Theorem A is existentially complete in $H / Q_{j}$. Thus $G / P_{j} \vDash \exists \mathbf{x} \phi\left(\mathbf{x}, \mathbf{g}+P_{j}\right)$. Let $\mathbf{f}_{j}$ be a solution in $G_{j}$, chosen to be 0 if $\mathbf{g} \in P_{j}$. Hence $\mathbf{f}=\left(\mathbf{f}_{j}\right)_{j \in I} \in G$ and $G \vDash \phi(\mathbf{f}, \mathbf{g})$. Thus $G$ is algebraically closed. Existential completeness fails as follows: Let $H=G \oplus G$. If $G$ is embedded in $H$ 
via $x \mapsto(x, x)$ then $G \subseteq H$. If $f=(g, g)$ for $0<g \in G$, then $H \vDash \exists x \exists y(x \wedge y=$ $0 \& x+y=f \& 0<x \& 0<y$ ) but this sentence is not satisfied in $G$.

In particular, 2 (the rationals) is algebraically closed but not existentially complete.

A positive element $g$ of an abelian $l$-group $G$ is said to be basic if $\{h \in G \mid$ $0 \leqslant h<g\}$ is totally ordered. Since every abelian lattice-ordered group is a subdirect product of totally ordered groups (namely $G \leqslant \Pi\{G / P \mid P$ a prime subgroup $\}$ ) then the proof of Proposition 4 shows the first part of the next corollary. The second part follows from the embedding $G \rightarrow \bar{G}$.

COROLlary 5. If $G$ is an existentially complete abelian lattice-ordered group, then $G$ has no basic elements and is divisible. Consequently $G$ is existentially complete in the class of torsion-free abelian groups.

Let $G \in \mathbb{Q}$ and $0<h \in G$. The convex $l$-subgroup of $G$ generated by $h$ is denoted by $G(h) . G(h)=\{f \in G:-n h \leqslant f \leqslant n h$ for some positive integer $n\}$. We now seek a first order sentence which is equivalent to $f \in G(h)$ when interpreted in any existentially complete member of $\mathbb{Q}$. Let $0<g, h \in G \in \mathbb{Q}, g$ splits over $h$ if

$$
\exists x_{0} \exists x_{1}\left(x_{0}+x_{1}=g \& x_{0} \wedge x_{1}=0 \& x_{0} \neq 0 \& x_{1} \neq 0 \& x_{0} \wedge h=0\right) \text {, }
$$

i.e., $g$ can be written as the sum of two strictly positive disjoint elements one of which is disjoint from $h$.

Lemma 6. Let $0<g, h \in G \in \mathbb{Q}$. Then $g \notin G(h)$ if and only if there is $H \in \mathbb{Q}$ such that $G \subseteq H$ and $g$ splits over $h$ in $H$.

Proof. If $g \notin G(h)$, choose a convex $l$-subgroup $P$ of $G$ maximal with respect to containing $G(h)$ but not $g$. Then $P$ is a prime subgroup of $G$ and $H=G \oplus G / P$ $\in \mathbb{Q}$. $l$-embed $G$ in $H$ via $f \mapsto(f, f+P) . g$ splits over $h$ in $H$ (take $x_{0}=(g, P)$ and $x_{1}=(0, g+P) \neq 0$ since $\left.g \notin P\right)$.

Conversely, if $g \in G(h)$ and $g$ splits over $h$, let $f_{0}, f_{1}>0$ be such that $f_{0} \wedge h=0$ $=f_{0} \wedge f_{1}$ and $f_{0}+f_{1}=g$. Now $g<n h$ for some positive integer $n$. Hence $0<f_{0}$ $=f_{0} \wedge g \leqslant f_{0} \wedge n h=0$ by Lemma 2 . This contradiction completes the proof of Lemma 6.

Corollary 7. Let $0<g, h \in G \in \mathbb{Q}$. If $G$ is existentially complete in $\mathcal{Q}$, then $g \in G(h)$ if and only if $g$ fails to split over $h$ in $G$.

Proof of Theorem B, Part 1 . Let $G$ be existentially complete in $\mathcal{Q}, \mathcal{U}$ be a nonprincipal ultrafilter on $\omega$ and $H=\Pi G / \mathcal{Q}$. To show $\mathcal{Q}$ has no model companion, it is enough to prove that $H$ is not existentially complete in $\mathcal{Q}$. Let $0<g \in G$, and let $f^{\sim}, h^{\sim} \in H$ be defined by $f^{\sim}=(g, 2 g, 3 g, \ldots)^{\sim}, h^{\sim}=$ $(g, g, g, \ldots) . f^{\sim}$ fails to split over $h \sim$ since it fails to do so at every coordinate, but $f^{\sim} \notin H\left(h^{\sim}\right)$ since $\mathscr{Q}$ is nonprincipal. Hence, by Corollary 7, $H$ is not existentially complete. So $\mathbb{Q}$ has no model companion.

Proof of Theorem B, Parts 2 And 3. Recall that $G \in \mathbb{Q}$ is Archimedean if

$$
\forall x_{0} \forall x_{1}\left(\underset{n=1}{\infty}\left(n x_{0} \leqslant x_{1}\right) \rightarrow x_{0}<0\right) \text {. }
$$


This is equivalent to $\forall x_{0} \forall x_{1}\left(x_{0}>0 \rightarrow\left(\exists x_{2}\right)\left(x_{2} \in G\left(x_{0}\right) \& x_{2} \nless x_{1}\right)\right)$ Replacing $x_{2} \in G\left(x_{0}\right)$ by " $x_{2}$ fails to split over $x_{0}$ "" we obtain a first order sentence $\phi$ which, by Corollary 7 , is $\forall \exists \forall$ and is equivalent, in existentially complete structures, to the Archimedean property. Specifically, $\phi$ is

$$
\begin{aligned}
& \forall x_{0} \forall x_{1} \exists x_{2} \forall x_{3} \forall x_{4}\left(x _ { 0 } \ngtr 0 \text { or } \left[x _ { 2 } \$ x _ { 1 } \& \left(0 \nless x_{3} \text { or } 0 \nless x_{4}\right.\right.\right. \text { or } \\
& \left.\left.\left.x_{3} \wedge x_{4} \neq 0 \text { or } x_{3}+x_{4} \neq x_{2} \text { or } x_{3} \wedge x_{0} \neq 0\right)\right]\right) \text {. }
\end{aligned}
$$

The proof of part 3 requires finding an Archimedean, existentially complete abelian l-group, which we now proceed to do.

Let $X$ be a topological space which is Hausdorff, not compact, perfect (no isolated points) and has a basis of compact clopen sets (e.g., $X$ is a disjoint union of a countable number of Cantor sets). Endow $\mathbf{R}$ with the discrete topology and consider $\bar{C}(X, \mathbf{R})$, the collection of continuous real valued functions from $X$ into $\mathbf{R}$ having compact support. $\bar{C}(X, \mathbf{R})$ is an abelian lattice-ordered group under addition of functions and the pointwise order $(f \leqslant g$ if and only if $f(x) \leqslant g(x)$ for all $x \in X$ ).

Note that $\bar{C}(X, \mathbf{R})$ is Archimedean.

THEOREM 8. $\bar{C}(X, \mathbf{R})$ is existentially complete in $\mathbb{Q}$.

Proof. Let $f \in \bar{C}=\bar{C}(X, \mathbf{R})$ and $S(f)=\{x \in X: f(x) \neq 0\}$. Then $S(f)$ is compact and equals $\cup_{0 \neq r \in \mathbf{R}} f^{-1}(r)$. Hence $S(f)=\bigcup_{i=1}^{n} f^{-1}\left(r_{i}\right)$ for some $r_{1}, \ldots, r_{n} \in \mathbf{R}$ so $f$ has finite range.

If $P$ is a prime subgroup of $\bar{C}$ and $P \neq \bar{C}$, let $T$ be a finite subset of $\bar{C}^{+} \backslash P=$ $\{f \in \bar{C} \backslash P: f \geqslant \underline{0}\}$. Then $\wedge T>0$. (If $\wedge T=0 \in P$, then $\min \{P+t: t \in T\}=$ $P$; i.e., some $t \in T$ belongs to $P$.) Hence $\cap_{g \in T} S(g) \neq \varnothing$. So $\bigcap_{g \in \bar{C}^{+} \backslash P} S(g) \neq \varnothing$. If $x \in \cap_{g \in \bar{C}^{+} \backslash P} S(g)$, then clearly $P_{x} \subseteq P$ where $P_{x}=\{f \in \bar{C}: f(x)=0\}$. But $P_{\underline{x}}$ is a maximal prime subgroup of $\bar{C}$ (see [1]) so the only prime subgroups of $\bar{C}$ are $\bar{C}$ and $P_{x}(x \in X)$.

Now let $\mathbf{g} \in \bar{C}, H \in \mathbb{Q}$ and $\bar{C} \subseteq H \vDash \exists \mathbf{u} \phi(\mathbf{u}, \mathbf{g})$, a primitive formula. $\phi(\mathbf{u}, \mathbf{g})$ is equivalent to $\bigwedge_{i=0}^{s-1} w_{i}(\mathbf{u}, \mathbf{g})=0 \& \bigwedge_{i=s}^{t} w_{i}(\mathbf{u}, \mathbf{g}) \neq 0$. Let $\psi(\mathbf{u}, \mathbf{g})$ be $\bigwedge_{i=0}^{s-1} w_{i}(\mathbf{u}, g)=0$ and $\theta_{i}(\mathbf{u}, \mathbf{g})$ be $\psi(\mathbf{u}, \mathbf{g}) \& w_{i}(\mathbf{u}, \mathbf{g}) \neq 0(s \leqslant i \leqslant t)$. Note that $\psi(\mathbf{u}, \mathbf{g})$ is a positive formula.

(I) Let $V$ be any compact open set on which $\mathbf{g}$ is constant. Then there is $\mathbf{r} \in \mathbf{R}$ such that $\mathbf{R} \vDash \psi(\mathbf{r}, \mathbf{g}(x))$ for all $x \in V$.

(II) For $s \leqslant i \leqslant t$, there is a nonempty compact open set $X_{i}$ and $\mathbf{r}^{\prime}(i) \in \mathbf{R}$ such that $g$ is constant on $X_{i}$ and $\mathbf{R} \vDash \theta_{i}\left(\mathbf{r}^{\prime}(i), \mathbf{g}(x)\right)$ for all $x \in X_{i}$.

To prove (I), let $x \in V$ and $Q$ be prime in $H$ such that $Q \cap \bar{C}=P_{x}$. Then $\mathbf{R} \cong \bar{C} / P_{x}=\bar{C} / \bar{C} \cap Q \cong\langle Q, \bar{C}\rangle / Q \subseteq H / Q \vDash \exists \mathbf{u} \psi(\mathbf{u}, \mathbf{g}(x))$ where $\mathbf{g}+Q$ is identified with $\mathbf{g}(x)$ in the indicated way. By Theorem $A$, there is an $\mathbf{r} \in \mathbf{R}$ such that $\mathbf{R} \vDash \psi(\mathbf{r}, \mathbf{g}(x))$. Since $\mathbf{g}$ is constant on $V$, this choice of $\mathbf{r} \in \mathbf{R}$ will work throughout $V$.

To prove (II), let $\mathbf{h} \in H$ be such that $H \vDash \theta_{i}(\mathbf{h}, \mathbf{g})$. Let $Q$ be a convex $l$-subgroup of $H$ maximal with respect to not containing $w_{i}(\mathbf{h}, \mathbf{g})$. Then $Q$ is prime, so $Q \cap \bar{C}=P_{x}$ for some $x \in X$ or $Q \cap \bar{C}=\bar{C}$. Note $H / Q \vDash \theta_{i}(\mathbf{h}+Q, \mathbf{g}+Q)$. In 
the first case, the proof continues the same as (I), since $\mathbf{R} \cong \bar{C} / P_{x} \subseteq H / Q$ F $\exists \mathbf{u} \theta_{i}(\mathbf{u}, \mathbf{g}(x)) ; X_{i}$ is any compact open set containing $x$ on which $\mathbf{g}$ is constant. In the second case, $Q \supseteq \bar{C}$ so $H / Q \vDash \theta_{i}(\mathbf{h}+Q, Q)$. Now $\mathbf{R} \vec{\oplus} H / Q \vDash \exists \mathbf{u} \theta_{i}(\mathbf{u}, \mathbf{0})$ and again we have by Theorem $A$ that $R \vDash \exists u \theta_{i}(\mathbf{u}, 0)$ (see two paragraphs hence for the definition of $\vec{\oplus})$. Pick $X_{i}$ any nonempty compact open set disjoint from $S(\mathbf{g})=$ $\{x \in X: \mathbf{g}(x) \neq 0\}(S(\mathbf{g})$ is a finite union of compact sets $S(g)$ so is compact, whereas $X$ is not; hence $X_{i}$ exists). Then $\mathbf{R} \vDash \theta_{i}\left(\mathbf{r}^{\prime}(i), \mathbf{g}(x)\right)$ for some $\mathbf{r}^{\prime}(i) \in R$ (all $x \in X_{i}$ ). This proves (II).

Now for $s \leqslant i \leqslant t$, let $X_{i}, \mathbf{r}^{\prime}(i)$ be given by (II). By the perfectness of $X$, we may assume $X_{i} \cap X_{j}=\varnothing$ if $i \neq j$. Let $Y=S(\mathrm{~g})$ and let $V_{1}, \ldots, V_{k}$ be a partition of $Y \backslash \cup_{i=s}^{t} X_{i}$ into compact open sets on each of which $\mathbf{g}$ is constant. By (I), $\mathbf{R} \vDash \psi(\mathbf{r}(i), \mathbf{g}(x))$ on $V_{i}$ for some $r(i) \in \mathbf{R}(1 \leqslant i \leqslant k)$. Let

$$
\mathbf{f}=\sum_{i=1}^{k} \mathbf{r}(i) c_{V_{i}}+\sum_{i=s}^{t} \mathbf{r}^{\prime}(i) c_{X_{i}} \quad \text { where } c_{V}(x)= \begin{cases}1 & \text { if } x \in V, \\ 0 & \text { if } x \notin V .\end{cases}
$$

Then $\mathbf{f} \in \bar{C}$ and $\bar{C} \vDash \phi(\mathbf{f}, \mathbf{g})$, so $\bar{C}$ is existentially complete in $Q$. This completes the proof of Theorem 8 .

Proof of Theorem B, Part 3. Here $\phi$ stands for the sentence constructed in the proof of part 2. Consider the group $\mathbf{Z} \oplus \mathbf{Z}$ (Z the group of integers) ordered by: $(a, b) \leqslant(c, d)$ if and only if $a<c$ or $(a=c \& b \leqslant d)$, the lexicographic ordering. Denote this $o$-group by $\mathbf{Z} \vec{\oplus} \mathbf{Z} . n(0,1) \leqslant(1,0)$ for all positive integers $n$, yet $(0,1)>0$. Hence if $G$ is existentially complete in $\mathcal{Q}$ and $G \supseteq \mathbf{Z} \vec{\oplus} \mathbf{Z}, G \vDash \neg \phi$. In particular, choose such a $G$ infinitely generic. Since $Q$ clearly satisfies the joint embedding property ( $A \oplus B \in \mathbb{Q}$ if $A, B \in \mathbb{Q}$ ), all infinitely generic models satisfy $\neg \phi$.

To complete the proof of the theorem, we must show $\varnothing \vDash \neg \neg \phi$. Throughout this paragraph, recall that $\neg \phi$ was an attempt to capture $\left(\exists x_{0}>0\right)\left(\exists x_{1}>0\right)\left(\forall x_{2}\right)$ $\left(x_{2} \in G\left(x_{0}\right) \rightarrow x_{2} \leqslant x_{1}\right)$. If $\varnothing$ does not force $\neg \neg \phi$, there are $c_{0}, c_{1} \in C$ and a condition $p \supseteq\left\{c_{0}>0, c_{1}>0\right\}$ such that

$$
\begin{aligned}
p \|\left(\forall x_{2}\right)\left[x _ { 2 } < c _ { 1 } \text { or } ( \exists x _ { 3 } > 0 ) ( \exists x _ { 4 } > 0 ) \left(x_{3}+x_{4}=x_{2} \& x_{3} \wedge x_{4}=0\right.\right. \\
\left.\left.\& x_{3} \wedge c_{0}=0\right)\right] .
\end{aligned}
$$

Let $\theta=\exists \mathbf{x} \bigwedge p(\mathbf{x})$ where $p(\mathbf{x})$ is obtained from $p$ by replacing each constant by a corresponding variable. Note that $\theta$ is existential. Since $p$ is consistent, $K \vDash \theta$ for some $K \in \mathbb{Q}$. Hence $K \oplus \bar{C}(X, \mathbf{R}) \vDash \theta$. By Theorem $8, \bar{C}(X, \mathbf{R}) \vDash \theta$. Since $\bar{C}(X, \mathbf{R})$ is Archimedean, there is a positive integer $n$ such that $\bar{C}(X, \mathbf{R}) \vDash \exists \mathbf{x}\left(\Lambda p(\mathbf{x}) \& n x_{0}\right.$ $\left.\$ x_{1}\right)$. Hence $p \cup\left\{n c_{0} \$ c_{1}\right\}$ is consistent. Let $c_{2}$ be a constant not occurring in $p$. Then $q=p \cup\left\{n c_{0} \$ c_{1}, c_{2}=n c_{0}\right\}$ is a condition extending $p$, so there is a condition $r \supseteq q$ such that $r \Vdash c_{2}<c_{1}$ or $\left(\exists x_{3}>0\right)\left(\exists x_{4}>0\right)\left(x_{3} \wedge x_{4}=0 \& x_{3}+\right.$ $\left.x_{4}=c_{2} \& x_{3} \wedge c_{0}=0\right)$. Thus $r \supseteq\left\{n c_{0} \$ c_{1}, c_{2}=n c_{0}, c_{2}<c_{1}\right\}$ or $r \supseteq\left\{n c_{0} \$ c_{1}\right.$, $\left.c_{2}=n c_{0}, c_{3}>0, c_{4}>0, c_{3} \wedge c_{4}=0, c_{3}+c_{4}=c_{2}, c_{3} \wedge c_{0}=0\right\}$ for some $c_{3}$, $c_{4} \in C$. The first is clearly impossible since $r$ is consistent and the second yields $c_{3} \wedge c_{0}=0$ and $0<c_{3}=c_{3} \wedge c_{2}=c_{3} \wedge n c_{0}$, contradicting Lemma 2. Hence, if $G$ is finitely generic, $G \vDash \phi$ and $G$ is accordingly Archimedean. 
$G \in \mathbb{Q}$ is said to be hyperarchimedean if every $l$-homomorphic image of $G$ is Archimedean. It is a stronger condition than Archimedean (the full Cartesian product of an infinite number of copies of $\mathbf{R}$ is Archimedean but not hyperarchimedean since the quotient by the direct sum is not Archimedean). It is equivalent to each of (i) For every $0<f, g \in G \in \mathbb{Q}$, there is a positive integer $n$ such that $n f \wedge g=(n+1) f \wedge g$; (ii) For every $0<f, g \in G \in \mathcal{Q}$, there are $g_{0}, g_{1} \in G$ such that $g=g_{0}+g_{1}, g_{0} \in G(f)$ and $g_{1} \wedge f=0$ (see [1]). Using these facts and the fact that $\bar{C}(X, \mathbf{R})$ is hyperarchimedean, it is straightforward to prove that any finitely generic abelian lattice-ordered group is hyperarchimedean (use (ii) and Corollary 7 to get a sentence $\theta$ which when interpreted in existentially complete models says hyperarchimedean). The details are included in [9].

We know of no example of an $\mathcal{Q}$-existentially complete model that is Archimedean but not hyperarchimedean. Also we would like to distinguish the finitely generic models among the hyperarchimedean existentially complete models of $\mathcal{Q}$. See [19] for a parallel in nilpotent class 2 groups.

We conclude this portion of the paper with the following fact:

Proposition 9. There are $2^{\aleph_{0}}$ countable pairwise nonisomorphic existentially complete abelian lattice-ordered groups.

Proof. Let $A$ be any set of $2^{\aleph_{0}}$ mutually algebraically independent irrational numbers, and consider, for each $a \in A, \mathbf{Z} \oplus \mathbf{Z} a$ ordered as a subgroup of $\mathbf{R}$. We claim that these subgroups are mutually nonisomorphic. Suppose $\mathbf{Z} \oplus \mathbf{Z} a \cong \mathbf{Z} \oplus$ $\mathbf{Z} b$ via the isomorphism $\boldsymbol{\theta}$. It is well known that any isomorphism between ordered subgroups of $\mathbf{R}$ is realized by multiplication by some positive $r \in \mathbf{R}$. Thus $1 \theta=1 r$ $=m+n b$ and $p+q b=a \theta=a r=a(m+n b)$ for some $m, n, p, q \in \mathbf{Z}$. But this implies algebraic dependence of $a$ and $b$. Thus there are $2^{\aleph_{0}}$ pairwise nonisomorphic 2-generator abelian lattice-ordered groups. Hence any countable abelian lattice-ordered group can accomodate only countably many of these groups. Since every countable abelian lattice-ordered group is contained in a countable existentially complete abelian lattice-ordered group, the proposition follows.

It is interesting to note that one can display $2^{\kappa_{0}}$ such groups. Let $X$ be a countable disjoint union of Cantor sets. $X$ has a countable Boolean algebra $\mathscr{B}$ of clopen sets. For each $a \in A$ let $G_{a}$ be the $l$-subgroup of $\bar{C}(X, \mathcal{2} \oplus \mathscr{2} a)$ consisting of those $f$ for which $f^{-1}(r) \in \mathscr{B}$ for all $r$. It is easy to show that $G_{a} \simeq G_{b}$ if and only if $\mathcal{2} \oplus \mathcal{Q} a \cong \mathscr{L} \oplus \mathcal{Q} b$ (as ordered subgroups of $\mathbf{R}$ ) and a repetition of the proof of Theorem 8 shows that $G_{a}$ is e.c.

2. Discrete $l$-groups. We now turn to some companionable theories. The theories under consideration all involve a distinguished "unit" 1 , named by a new constant adjoined to the language of abelian lattice-ordered groups. Consequently any homomorphism must preserve this unit.

An abelian o-groups is discrete if it has a smallest positive element-equivalently, if it has a smallest convex subgroup isomorphic to $\mathbf{Z}$. We shall identify this convex subgroup with $\mathbf{Z}$. Let $\mathscr{D}$ denote the theory of discrete abelian $o$-groups: we need 
only adjoin to the theory of abelian o-groups the axiom stating that 1 is the smallest positive element. We call 1 the unit of the group.

A discrete abelian $o$-group is said to be regularly discrete if $|G / p G|=p$ for every prime number $p$. The class $\mathscr{D}^{*}$ of regularly discrete abelian $o$-groups is elementary (say that, for each prime $p$, every element is congruent modulo $p$ to one of $0,1, \ldots, p-1)$. Robinson and Zakon [16] showed that $\mathscr{D}^{*}$ is complete and model-complete, and Conrad [4] observed that $G$ is regularly discrete if and only if $G / \mathbf{Z}$ is divisible ( $G$ abelian).

THEOREM 10. $\mathscr{D} *$ is the model companion, but not the model completion, of $\mathscr{D}$.

The proof follows from the next two lemmas.

LEMMA 11. Every discrete abelian o-group is embeddable in a regularly discrete abelian o-group.

Proof. It suffices to prove the following: If $G \vDash \mathscr{D}, g \in G$ and $p$ is a prime number, then $G$ is embeddable in some $H \vDash \mathscr{D}$ in which $g$ is congruent modulo $p$ to one of $0,1,2, \ldots, p-1$. For each $i, 0 \leqslant i<p$, let $H_{i}$ be the subgroup of the divisible hull of $G$ generated by $G$ together with $(g-i) / p$. It will suffice to show that 1 remains the smallest positive element of some $H_{i}$. If not, each $H_{i}$ contains an element strictly between 0 and 1 . But there are only $p-1$ such elements in the divisible hull of $G$; hence two of them are equal. Thus there are integers $i, j, n_{i}, n_{j}$ such that $0<n_{i}<p, 0<n_{j}<p, 0 \leqslant i<j<p$ and there are $g_{i}, g_{j} \in G$ such that

$$
g_{i}+n_{i}(g-i) / p=g_{j}+n_{j}(g-j) / p
$$

whence, in $G$,

$$
\left(n_{j}-n_{i}\right) g+n_{i} i-n_{j} j \equiv 0 \quad(\bmod p)
$$

Now $n_{i}$ and $n_{j}$ are necessarily distinct, so $n_{i}-n_{j}$ is relatively prime to $p$. By elementary number theory, this implies that, in $G$,

$$
g \equiv r\left(n_{i} i-n_{j} j\right) \quad(\bmod p)
$$

for some integer $r$ and hence $g \equiv i_{0}(\bmod p)$ for some $0<i_{0}<p$. Thus we simply set $G=H$.

LEMMA 12. OD does not have the amalgamation property.

Proof. Let $G$ be the subgroup of $\mathcal{Q} \vec{\oplus} \mathcal{Q}$ generated by $\left\{\left(k / p_{k}, 1 / p_{k}\right): p_{k}\right.$ is the $k$ th prime number $\}$. $G$ is discrete with smallest positive element $1=(0,1)=$ $3(2 / 3,1 / 3)-2(1 / 2,1 / 2)[5$, p. 4.9]. Let $a=(1 / 2,1 / 2)$, and let $H$ and $K$ be the subgroups of $2 \vec{\oplus} \mathcal{Q}$ generated by adjoining to $G$ respectively $a / 2$ and $(a-1) / 2$. $H$ and $K$ are discrete with smallest positive element 1 ; if, for example, this were false for $H$ then $(0,1 / 2) \in H$, since $2 H \subseteq G$. This implies that

$$
(0,1 / 2)=m a / 2+\sum_{k} m_{k}\left(k / p_{k}, 1 / p_{k}\right)
$$

for some finite collection of integers $m, m_{1}, m_{2}, \ldots$ Setting second components equal results in the equation 


$$
(2-m) \prod_{k} p_{k}=4 \sum_{k} m_{k} \prod_{i \neq k} p_{i}
$$

whence $m$ is even. But this puts $(0,1 / 2) \in G$, a contradiction.

Therefore the inclusion maps $G \rightarrow H, G \rightarrow K$ are embeddings, but this amalgam cannot be completed in $\mathscr{D}$. For if it were, then, in the amalgamating group, both $a$ and $a-1$ would be divisible by 2 , which is impossible.

Our next example exploits the extremely strong parallels between the class of subdirect products of discrete $o$-groups and the class of semiprime commutative rings with identity. Indeed, these results were inspired by reading the ring-theoretic results of Lipshitz and Saracino [11], and later Macintyre [12] and Dauns-Hoffmann [6]. We present a generalization of Macintyre's result and show how it can be applied to the above class of lattice-ordered groups. See Remark 5 in $\$ 3$ for comments on the relation between these results and the existing literature; also the footnote to Theorem 21 .

Let $\mathscr{D} \mathcal{E}$ be the class of subdirect products of discrete abelian 0 -groups. Such groups are $l$-groups under pointwise order, so we consider $\mathscr{D} \mathcal{L}$ within the language of $l$-groups with new constant 1 (call such abelian $l$-groups discrete). It is well known that $\mathscr{D} \mathcal{L}$ is an elementary class, being axiomatized by the set of all universal Horn consequences of $\mathscr{D}$. But here an explicit axiom set can be displayed. First some preliminaries from the theory of $l$-groups, all of which can be found in [1].

Let $G$ be an abelian l-group. An element $u \in G$ is a weak order-unit if $u \wedge x=0$ only when $x=0$, and $s \in G$ is singular if $0<s$ and $0<x<s$ always implies $x \wedge(s-x)=0$.

LEMMA 13. (1) $A$ weak order-unit is a member of no minimal prime subgroup.

(2) Every prime subgroup $P$ not containing a given singular element $s$ is minimal; in this case $G / P$ is a discrete ordered group with unit $s+P$.

Proof. Proposition 3.4.13 of [1] states that if $u$ is a member of a prime subgroup $P$ then $x \wedge u=0$ for some $x \notin P$, hence $u$ cannot be a weak order unit. Part 2 follows from Proposition 11.2.8 and Corollary 11.2.11 of [1].

It is important to observe that, in a subdirect product of discrete ordered groups, singular elements are precisely the functions taking only 0 or 1 as values.

Proposition 14. $\mathscr{D} \mathcal{E}$ is axiomatized by the axioms for abelian l-groups, together with the formula stating that 1 is a singular, weak order-unit.

Proof. If $G \in \mathscr{D} \mathcal{E}$ then the interpretation of 1 in $G$ must be singular and a weak order-unit. Conversely, Lemma 13 shows that the representation $G<$ $\Pi\{G / P: P$ a minimal prime subgroup $\}$ puts $G \in \mathscr{D} \mathcal{L}$.

Let $\mathscr{Q} \mathcal{L}^{*}$ be the theory got by adjoining to $\mathscr{Q} \mathcal{L}$ the axioms:

(1) There are no minimal singular elements;

(2) For each prime number $p, G$ is divisible by $p$ modulo the singular elements of $G$; more precisely, every element is congruent $\bmod p$ to a sum of at most $p$ singular elements; 
(3) For every positive $g$ and singular $s$ there are $x, y$ such that $g=x+y$, $x \wedge y=0, x \wedge s=0$, and $y \wedge(1-s)=0$. (Terminology: $g$ splits over $s$.)

Axiom (2) implies that models of $\mathscr{D}^{*}$ are subdirect products of regularly discrete abelian o-groups. From this viewpoint, axiom (3) says that for every positive $g$ and singular $s$ there is some $y$ equal to $g$ on those factors where $s$ is 1 , and equal to 0 elsewhere.

THEOREM 15. $\mathscr{D} \mathcal{L}^{*}$ is the model companion but not the model completion of $\mathscr{D} \mathscr{L}$.

The latter is a consequence of Lemma 12, which also shows that $\mathscr{D} \mathcal{L}$ does not have the amalgamation property.

Model consistency follows from the easily proven fact that $\bar{C}(X, D) \vDash \mathscr{D} \mathcal{L}^{*}$, where $\bar{C}(X, D)$ is the l-group of continuous functions from a compact, totally disconnected, Hausdorff space $X$ without isolated points to a regularly discrete abelian $o$-group $D$ (where $D$ is furnished with the discrete topology). If $G \vDash \mathscr{D} \mathcal{L}$, $G \leqslant \Pi G / P$ where $P$ ranges over all minimal prime subgroups of $G$. For each $P$ embed $G / P$ in a regularly discrete ordered group $D_{P}$. We have then $G<\Pi G / P$ $\leqslant \Pi D_{P} \leqslant \Pi \bar{C}\left(X, D_{P}\right)$ for $X$ as above. Since $\mathscr{D}^{L^{*}}$ is evidently preserved under products, model-consistency is proven.

Model-completeness can be proved either by the Lipshitz-Saracino method as given by Cherlin [2] or by Macintyre's sheaf-theoretic attack [12]. We employ the latter and now give the relevant definitions. ${ }^{2}$

Let $\mathcal{L}$ be a first-order language. To simplify the definition, we assume that $\mathcal{L}$ has no relation symbols. A sheaf of $\mathcal{L}$-structures is a triple $\langle S, \pi, X\rangle$ where

(i) $S$ and $X$ are topological spaces;

(ii) $\pi$ maps $S$ onto $X$ so that each point in $S$ has a neighborhood which is mapped homeomorphically onto an open set in $X$;

(iii) each "stalk" $S_{x}=\pi^{-1}(x)$ is an $\mathcal{L}$-structure on which the operations of $\mathcal{L}$ are continuous relative to the topology of $S$;

(iv) for each constant $a$ of $\mathcal{E}$ the function $\hat{a}$ assigning to each $x$ the interpretation of $a$ in $S_{x}$ is continuous.

A section of a sheaf is any continuous $f: X \rightarrow S$ for which $\pi \circ f$ is the identity on $X$. The set of all sections is an $\mathcal{E}$-structure, under the obvious pointwise operations, and is denoted by $\Gamma(X, S)$.

\footnotetext{
${ }^{2}$ Since submitting this paper, it has come to our attention that the model-completeness of $\mathscr{D} *$ can be deduced directly from our Lemma 20 and results appearing in the existing literature. Specifically, if $|x|=x \vee(-x)$ and $\chi(x, y)=|x-y| \wedge 1$, then $\tau(x, y, z, w) \equiv[|w-z| \wedge(1-\chi(x, y))] \vee[|w-x| \wedge$ $\chi(x, y)]=0$ is a discriminator formula for discrete 0 -groups so $\operatorname{Th}(\mathcal{C})$ is model-complete (where $\mathcal{C}$ is the class of all sections $\Gamma(X, S)$ as $X$ ranges over compact, totally disconnected Hausdorff spaces without isolated points and $S$ ranges over sheaves of members of $\mathscr{D}$ *. (See $\$ 10$ of Stanley Burris and Heinrich Werner, Sheaf constructions and their elementary properties, Trans. Amer. Math. Soc. 248 (1979), 269-309, for the relevant definition and proof.)) Alternatively, a proof that $\mathrm{Th}(\mathcal{C})$ is model-complete can be given along the same lines as V. Weispfenning, Model theory of lattice products, Habilitationschrift, Universität Heidelberg, 1978 (887.11-7.13) by replacing divisible ordered abelian groups as a stalk theory by regular discrete abelian o-groups and letting the weak projector of $a$ be $a^{\sim}=1-(|a| \wedge 1)$. We are most grateful to the authors for pointing this out to us and have retained our original approach only for those who wish for a more mundane one.
} 
Call $\operatorname{Th}(\Gamma(S, X))$ the section theory and $\operatorname{Th}\left(\left\{S_{x} \mid x \in X\right\}\right)$ the stalk theory of the sheaf.

Macintyre gave an answer to the question: If the stalk theory is model-complete, under what conditions is the section theory model-complete? His conditions involve the theory of rings. Comer [3] provided more general conditions; but neither of these fits our situation. However, a careful reading of the proof of Macintyre's Theorem 2 [12] shows it to be true in a much more general context, displayed below.

A theory is positively model-complete if it is model-complete and every existential formula is equivalent to a positive existential formula.

Let $(S, \pi, X)$ be a sheaf of $\mathcal{L}$-structures. For a formula $\phi(v)$ and $\mathrm{f} \in \Gamma(X, S)$ define

$$
K_{\phi}(\mathbf{f})=\left\{x \in X \mid S_{x} \vDash \phi(\mathbf{f}(x))\right\}
$$

Consider the following conditions:

(A) $X$ is a compact, totally disconnected Hausdorff space without isolated points;

(B) The stalk theory is positively model-complete;

(C) There is a 1-1 correspondence $d \mapsto \vartheta_{d}$ between some $\Delta \subseteq \Gamma(X, S)$ and the set of all clopen subsets of $X$;

(D) There is an existential formula $\Delta(v)$ defining $\Delta$ in $\Gamma(X, S)$.

(E) There are existential $\Theta_{n}\left(v_{0}, \ldots, v_{n}\right), \Psi(v)$, and $\Phi(u, v, w)$ such that in the section theory for all $d, \mathrm{~d} \in \Delta$ and all $f, g \in \Gamma(X, S), \Theta_{n}(\mathrm{~d}), \Psi(d)$, and $\Phi(f, g, d)$ hold respectively if and only if $X=\theta_{d_{0}} \cup \cdots \cup \vartheta_{d_{n}}, \theta_{d} \neq \varnothing$, and $f \uparrow \theta_{d}=$ $g \uparrow \theta_{d}$.

THEOREM 16. If the stalk theory is model-complete and (A)-(E) hold, then the section theory is model-complete.

The proof is the appropriate modification of the proof of Macintyre's Theorem 3 and is obvious. Macintyre's Theorem 2 can also be adapted to this situation:

THEOREM 17. Suppose $\mathcal{E}$ has relation symbols. If the stalk theory is complete and model-complete and (A)-(E) hold, provided that $\Psi$ is quantifier-free, then the section theory is model-complete.

Macintyre's Theorems 4 and 5 also generalize. We state the one we will use, his Theorem 5, which follows immediately from Theorem 16.

THEOREM 18. Let $T$ be a positively model-complete theory. Let $\mathcal{C}$ be the class of all $\Gamma(X, S)$ where $S$ is a sheaf of models of $T$, and $X$ is a compact, totally disconnected Hausdorff space without isolated points. If there are existential $\Delta, \theta_{n}, \Psi$, and $\Theta$ satisfying (D) and (E) for all $\Gamma(X, S) \in \mathcal{C}$, then $\mathrm{Th}(\mathcal{C})$ is model-complete.

COROLlARY 19. If $T$ is the theory of regularly discrete abelian o-groups (in the language with $0,1,+, \vee, \wedge$ ) then $\mathrm{Th}(\mathcal{C})$ is model-complete. 
Proof. First, $T$ is positively model-complete since, modulo $T, a \neq b$ is equivalent to $|a-b| \wedge 1=1$ (where $|x|=x \vee-x$ ). For (C) let $\Delta$ be the set of all singular elements of $\Gamma(X, S)$, the correspondence being given by $s \mapsto\{x \mid s(x)=1\}$. For (D) let $\Delta(v)$ be $v \wedge(1-v)=0$, and for (E) let $\Theta_{n}\left(v_{0}, \ldots, v_{n}\right)$ be $1=\bigvee_{i=0}^{n} v_{i}$, let $\Psi(v)$ be $v \neq 0$, and let $\Phi(u, v, w)$ be $|u-v| \wedge w=0$.

The proof of Theorem 15 is finished once we show that $\mathscr{D} \mathcal{L}^{*}=\operatorname{Th}(\mathcal{C})$. Clearly, $\operatorname{Th}(\mathcal{C}) \subseteq \mathscr{D} \mathcal{L}^{*}$ so it remains to prove

LEMMA 20. Every $G \in \mathscr{Q} \mathcal{L}^{*}$ is isomorphic to the lattice-ordered group of sections of some sheaf $\langle S, \pi, X\rangle$ of regularly discrete abelian o-groups for $X$ as above.

The proof is the analogue of Dauns and Hoffmann [6], outlined as follows: $M(G)$, the set of all minimal prime subgroups of $G$, when endowed with the hull-kernel topology (i.e., the basic open sets are $\theta_{g}=\{P \in M(G): g \notin P\}$ ) becomes a compact totally disconnected Hausdorff space without isolated points; in fact every $\theta_{g}$ is clopen. We define $S(G)$ to be the union of all $G / P, P \in M(G)$ and define $\pi: S(G) \rightarrow M(G)$ in the obvious way. Let $g \mapsto \hat{g}$ denote the canonical map $G \rightarrow \Pi\{G / P \mid P \in M(G)\}$. Then the set $\{\hat{g}(U): U$ clopen in $X\}$ is a basis for a topology on $S(G)$ making $\langle S, \pi, X\rangle$ into a sheaf of the desired kind, in which $g \mapsto \hat{g}$ is an isomorphism of $G$ into $\Gamma(X, S)$. Furthermore, the clopen subsets of $X$ are in 1-1 correspondence with the singular elements, these being precisely the characteristic functions of clopen sets. For any section $f$ there are $g_{1}, \ldots, g_{n} \in G$ and disjoint clopen $W_{1}, \ldots, W_{n}$ covering $X$ so that $f\left|W_{i}=\hat{g}_{i}\right| W_{i}$. By the splitting property (axiom 3 of $\mathscr{D L}^{*}$ ), we can assume $\hat{g}_{i}$ is zero off $W_{i}$, whence $f=\hat{g}_{1}$ $+\cdots+\hat{g}_{n}$, and so $\hat{G}$ is all of $\Gamma(X, S)$, as desired.

Inspecting the proof of Lemma 12, we observe that the failure of $\mathscr{D}$ and $\mathscr{D} \mathcal{L}$ to have the amalgamation property follows from the failure of certain groups to be divisible modulo their singular elements (i.e., axiom 2 fails). In fact, the reverse is also true in a sense. Define $\mathscr{D} \mathcal{E}^{\prime}$ to be the theory $\mathscr{D} \mathcal{L}$ with axiom 2 adjoined.

THEOREM 21. $\mathscr{D} *$ and $\mathbf{R}^{\mathscr{D}}$ ' have the amalgamation property, and therefore $\mathscr{D} \mathcal{L} *$ is the model completion of $\mathscr{D} \mathcal{L}^{\prime}$.

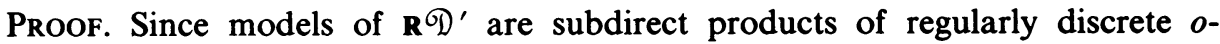
groups and conversely, it suffices to show that $\mathscr{D} *$ has the amalgamation property. Suppose $G, H, K \vDash \mathscr{D} *$ and $G \leqslant H, G \leqslant K$. Evidently $G / \mathbf{Z} \leqslant H / \mathbf{Z}$ and $G / \mathbf{Z}<$ $K / \mathbf{Z}$. Since the theory of abelian $o$-groups has the amalgamation property [14], there is an abelian $o$-group $L$ and embeddings (forgetting about 1 temporarily) $\eta_{1}$ : $H / Z \rightarrow L$ and $\eta_{2}: K / \mathbf{Z} \rightarrow L$ agreeing on $G / \mathbf{Z}$. By Hahn's embedding theorem there are embeddings $H \rightarrow H / \mathbf{Z} \vec{\oplus} \mathcal{Q}$ and $K \rightarrow K / \mathbf{Z} \vec{\oplus} \mathcal{Q}$ where one can assume $1 \mapsto(0,1)$. Let these embeddings be represented by $h \mapsto\left(h+Z, h \sigma_{1}\right), k \mapsto(k+$ $\left.\mathrm{Z}, k \sigma_{2}\right)$. Since $G$ is a pure subgroup of $H$ by model-completeness, $H / G$ is torsion-free and so can be totally ordered. Let $M=L \vec{\oplus} H / G \vec{\oplus} \mathcal{Q}$ and embed $H$ and $K$ in $M$ via

$$
h \mapsto\left((h+\mathbf{Z}) \eta_{1}, h+G, h \sigma_{1}\right), \quad k \mapsto\left((k+\mathbf{Z}) \eta_{2}, 0, k \sigma_{2}\right) .
$$

One routinely verifies that these embeddings agree on $G$ and that the subgroup of 
$M$ generated by the images of $H$ and $K$ is discrete with unit $(0,0,1)$, and hence the amalgamation has taken place in $\mathscr{D}$. Since $\mathscr{D}$ and $\mathscr{D}^{*}$ are mutually model consistent, this finishes the proof. ${ }^{3}$

Corollary 22. $\mathscr{D} \mathcal{L}^{*}$ is complete and decidable.

Proof. Since $\mathbf{Z} \in \mathscr{D} \mathcal{L}^{\prime}$ and is canonically contained in every member of $\mathscr{D} \mathscr{L}^{*}$, the amalgamation property for $\mathscr{D} \mathcal{L}^{\prime}$ together with the mutual model consistency of $\mathscr{D} \mathscr{L}^{\prime}$ and $\mathscr{D} \mathscr{L}^{*}$ imply that $\mathscr{D} \mathscr{L}^{*}$ enjoys the joint embedding property. Since $\mathscr{D} \mathcal{L}^{*}$ is model-complete, this ensures that it is complete. It now follows that it is decidable (since it is recursively axiomatizable).

THEOREM 23. Neither $\mathscr{D} *$ nor $\mathscr{D} e^{*}$ has elimination of quantifiers.

Proof. It is well known [13, Lemma 12] that this follows from $\mathscr{D}^{*}$ and $\mathscr{D}^{\mathcal{L}^{*}}$ being model companions, but not model completions, of universal theories.

3. Remarks and conjectures. (1) Is every finitely generic abelian lattice-ordered group a group of all real-valued functions with finite range? (Every hyperarchimedean $l$-group is a group of real-valued functions with finite range [1, Proposition 14.1.7].)

(2) Concerning the revised Wheeler conjecture: Theorem 15 shows that stability is not necessary (which was known-see [13]).

(3) We want this conjecture to read: If $T$ is companionable and ... then $T_{\mathrm{UH}}$ is companionable. In light of Theorems 16-18 we wonder if there are syntactical formulations of these sheaf-theoretic results which will make the conjecture true.

(4) In the class of existentially complete abelian lattice-ordered groups, is the property " $x \in\langle y\rangle$, the cyclic subgroup generated by $y$ " first-order-definable? We have proven this true in the class of existentially complete (non-abelian) latticeordered groups [9].

(5) $\$ 10$ of Burris and Werner (op. cit.) may well provide the correct framework to answer (3) and (4). Theorems 15 and 21 are very similar to the results obtained by Weispfenning (op. cit. or The model-completion of a class of lattice-ordered abelian groups, Notices Amer. Math. Soc. 23 (1976), p. A-349, Abstract \#76T-A75).

Acknowledgements. The results of this paper were first announced at the Ordered Group Conference in Boise, Idaho, in October, 1978. Statements of the theorems appear in the Proceedings of that conference [9]. The proof of Theorem B was first presented in detail at the Michigan-Ohio Logic Seminar in Autumn, 1978.

We are delighted to acknowledge the help and enthusiasm we have had from

\footnotetext{
${ }^{3}$ An alternative proof that $\mathscr{D} \mathcal{L}^{\prime}$ has the amalgamation property can be deduced as follows: By Pressburger (1929), the theory of regular discrete abelian o-groups allows a primitive recursive elimination of quantifiers, if one includes all congruences $\equiv_{n}$ with defining axioms in the language. Hence, by the transfer theorems of Weispfenning (op. cit.), so does $\mathscr{D} \mathcal{L}$. Thus $\mathscr{D} \mathcal{L}$ with the defining axioms for $\equiv_{n}(n \in \omega)$ has the amalgamation property. Since in $\mathscr{D} \mathcal{L}^{\prime}$, the congruences $\equiv_{n}$ are universally as well as existentially definable, these congruences are preserved under extensions and restrictions between $\mathscr{D} \mathcal{L}^{\prime}$ models. Therefore, $\mathscr{D} \mathcal{L}^{\prime}$ has the amalgamation property. This proof is due to Weispfenning to whom the authors are grateful.
} 
several colleagues. Specifically, we wish to thank Yuri Gurevich for several perspicuous discussions and Carol Wood for making us aware of much of the literature on applications of forcing to algebra. The whole paper was directed by that awareness. We are also extremely grateful to Stanley Burris and Volker Weispfenning for making us aware of their work on general Boolean products. These considerably shorten the proof of Theoem 15 (see the relevant footnotes).

This paper is dedicated to the memory of Abraham Robinson. Not only was he responsible for Theorem $\mathrm{A}$, but also for the pioneer work in model-theoretic forcing without which none of this research would have been possible. We would like to believe that, because of his interests, he would have enjoyed the results.

The first author wishes to acknowledge the support of the Bowling Green State University Faculty Research Committee.

\section{BIBLIOGRAPHY}

1. A. Bigard, K. Keimel and S. Wolfenstein, Groupes et anneaux réticulés, Lecture Notes in Math., vol. 608, Springer, Berlin and New York, 1977.

2. G. Cherlin, Model-theoretic algebra-selected topics, Lecture Notes in Math., vol. 521, Springer, Berlin and New York, 1976.

3. S. D. Comer, Complete and model-complete theories of monadic algebras, Colloq. Math. 34 (1976), $183-190$.

4. P. F. Conrad, Regularly ordered groups, Proc. Amer. Math. Soc. 13 (1962), 726-731.

5. __ Lattice-ordered groups, Tulane Lecture Notes, Tulane Univ., New Orleans, 1970.

6. J. Dauns and K. H. Hoffmann, The representation of biregular rings by sheaves, Math. Z. 91 (1966), $103-123$.

7. P. C. Eklof and G. Sabbagh, Model-completions and modules, Ann. Math. Logic 2 (1971), 251-295.

8. George Eliot, Middlemarch, 1872.

9. A. M. W. Glass and K. R. Pierce, Equations and inequations in lattice-ordered groups (Proc. Ordered Groups Conference, Boise, Idaho, 1978), Dekker, New York (to appear).

10. J. Hirschfeld and W. H. Wheeler, Forcing, arithmetic, division rings, Lecture Notes in Math., vol. 454, Springer, Berlin and New York, 1975.

11. L. Lipshitz and D. Saracino, The model companion of the theory of commutative rings without nilpotent elements, Proc. Amer. Math. Soc. 38 (1973), 381-387.

12. A. Macintyre, Model-completeness for sheaves of structures, Fund. Math. 81 (1973), 73-89.

13. __ Model-completeness, handbook of mathematical logic, (K. J. Barwise, editor), North-Holland, Amsterdam, 1977.

14. K. R. Pierce, Amalgamations of lattice-ordered groups, Trans. Amer. Math. Soc. 172 (1972), $249-260$.

15. A. Robinson, Complete theories, North-Holland, Amsterdam, 1956.

16. A. Robinson and E. Zakon, Elementary properties of ordered abelian groups, Trans. Amer. Math. Soc. 96 (1960), 222-236.

17. D. Saracino, Existentially complete nilpotent groups, Israel J. Math. 25 (1976), 241-248.

18. __ Existentially complete torsion-free nilpotent groups, J. Symbolic Logic 43 (1978), 126-134.

19. D. Saracino and C. Wood, Periodic existentially closed nilpotent groups, J. Algebra 58 (1979), 189-207.

20. W. H. Wheeler, A characterization of companionable, universal theories, J. Symbolic Logic 43 (1978), 402-429.

Department of Mathematics, Bowling Green State University, Bowling Green, Ohio 43403

Department of Mathematics, University of Missouri, Columbia, Missouri 65211 